\title{
Prehospital Advanced Cardiac Life Support with a Smartphone-Based Direct Medical Oversight in a Metropolitan City, Korea
}

\author{
Seung Hoon Jeon ${ }^{1}$, and Yongjin Park ${ }^{1, *}$ \\ ${ }^{1}$ Department of Emergency Medicine, College of Medicine, Chosun University, Gwangju, Republic of Korea
}

* Corresponding author: Yongjin Park, Department of Emergency Medicine, College of Medicine, Chosun University, 365 Philmun-daero, Dong-gu, Gwangju, Republic of Korea. Tel: +82622203285; Fax: +82622243501; Email: eryongjin@chosun.ac.kr

Received 2021 April 03; Revised 2021 May 01; Accepted 2021 May 18.

\begin{abstract}
Background: Out-of-hospital cardiac arrest is considered an important health care problem since it causes family breakdown and enormous social loss due to sudden death. Despite the efforts of many medical policy makers, paramedics, and doctors, the survival rate after cardiac arrest is only marginally increasing.

Objectives: This study aimed to determine whether advanced life support (ALS) under physician's direct medical oversight during an emergency through video call on smartphones was associated with improved out-of-hospital cardiac arrest (OHCA) outcomes on the "Smart Advanced Life Support (SALS)" pilot project.

Methods: This study was conducted using a "Before-After" controlled trial. The OHCA patients were divided into two periods in a metropolitan city. The basic life support (BLS group) and ALS using video calls on smartphones (SALS group) were performed in the 'Before' and 'After' phases in 2014 and 2015, respectively. The OHCA patients over 18 years of age were included in this study. On the other hand, the patients with trauma, poisoning, and family's unwillingness, as well as those who received no resuscitation were excluded from the study. The primary and secondary outcomes were survival to discharge and a good neurological outcome (cerebral performance category [CPC] 1-2), respectively. A propensity score matching was conducted to equalize potential prognostic factors in both groups. The adjusted odds ratio (OR) and 95\% confidence interval (95\% CI) were calculated for survival to discharge and good neurological outcome. Results: In total, 235 and 198 OHCA patients were enrolled in the BLS and the SALS groups, respectively. The outcomes were better in the SALS group, compared to the BLS group regarding the survival to discharge $(9.8 \%$ vs. $6.8 \%, \mathrm{P}<0.001)$ and good neurological outcome (6.6\% vs. $4.0 \%, \mathrm{P}<0.001)$, respectively. Regarding propensity score matching, 304 cases were randomly assigned to the SALS and BLS groups. The survivals to discharge rates after matching were $9.2 \%$ and $7.2 \%$ in the SALS and the BLS groups, respectively (P=0.06). Furthermore, the good neurological outcome rate was $5.9 \%$ in the SALS group versus 3.9\% in the BLS group (p=0.008). The adjusted ORs of the SALS group were estimated at 1.33 (95\% CI: $1.00-1.77$ ) for survival to discharge and 1.73 (95\% CI: $1.19-2.53$ ) for the good neurologic outcome, compared to those in the BLS group.

Conclusion: An emergency medical system intervention using the SALS protocol was associated with a significant increase in prehospital ROSC and improved survival and neurologic outcome after OHCA.
\end{abstract}

Keywords: Advanced cardiac life support, Emergency medical system, Out-of-hospital cardiac arrest, Smartphone

\section{Background}

In Korea, the recent standardized incidence rates of out-of-hospital cardiac arrest (OHCA) was 44.4 per 100,000 persons $(24,442$ persons experienced OHCA in 2009), which is similar to that reported in other countries (1). This includes a rise of more than $10 \%$, compared to 50.5 per 100,000 people in 2006 , which is increasing every year (2). However, despite the efforts of many medical policy officers, paramedics, and doctors, the survival outcomes after cardiac arrest remained at $4.8 \%$ in both 2013 and 2014, which was relatively poor, compared to other countries (3-5). Among them, it was seen that faster return of spontaneous circulation (ROSC) led to higher chances of good neurological outcome, and for this, it was considered to be more advantageous to make a pre-hospital ROSC. However, given the procedure in Korea for OHCA, only basic life support (BLS) is given at the site within 5 minutes, and the patient is then transported to the hospital; therefore, it is difficult to expect circulation recovery before arriving at a hospital.

A number of studies revealed that prehospital advanced life support (ALS) was not effective for OHCA patients (6-10). The experience of the paramedics exposed to the OHCA patients affects the treatment of cardiac arrest; however, the actual exposure of the paramedics is rare and declining. In the prehospital emergency medical services (EMS), the physician presence is associated with improved survival outcomes of the patient; nonetheless, the characteristics of the EMS operating in each country are different, and there are places where the operation is not possible in reality (11-13).

However, recently developed video communication technologies have created opportunities to make various attempts in the medical field. A "Smart Advanced Life Support" (SALS) pilot project was introduced to some areas on a trial basis, where in the event of a cardiac arrest, the nearest two units of the EMS are dispatched, and through the visual directions of an emergency physician, the paramedics will use the manual defibrillator, administer drugs, 
and perform advanced life support.

\section{Objectives}

This study aimed to determine whether SALS under physician's direct medical oversight through video call on smartphones was associated with improved OHCA outcomes in a metropolitan city.

\section{Methods}

\subsection{Design}

This study was conducted using a "Before-After" controlled trial. The OHCA patients in the study area were divided into two periods of "Before and After". The "Before" phase performed the same BLS for 12 months (January 2014 to December 2014), and the "After" phase performed the video medical guidance for a total of 12 months (January 2015 to December 2015).

In both periods, the emergency medical technician (EMT) filled out the patient's ambulance run sheet after being dispatched to the cardiac arrest scene; moreover, the ambulance run sheets were electronically stored in each provincial EMS headquarters managed by the National Fire Agency (NFA). In the "After" phase, the EMT additionally filled out the "SALS chart" directing physicians made the "Oversight physician report". Trained reviewers visited the hospital where the patient was transferred and reviewed the medical records to complete the outcomes according to the Utstein style (14). These data were electronically stored in the national emergency medical center (NEMC). Finally, the NEMC secured the sheet with the cooperation of the NFA and constructed the SALS database. Physicians responsible for database quality control in this study performed a structured and explicit data review. This study was practically impossible to obtain the informed consent of the subjects; accordingly, written consent was obtained from them. This study was carried after the Institutional Review Board's approval from the Research Ethics Committee of the Chosun University Hospital, Gwangju, South Korea (No. 2015-12-009).

\subsection{Setting}

The setting was a metropolitan city of the Republic of Korea with a population of approximately 1.5 million people in $501 \mathrm{~km}^{2}$, consisting mostly of urban areas. There are 30 ambulances, 270 paramedics, and 24 ambulance stations. Korean EMS has recently established a centrally based and twotiered system for OHCA patients, as well as two or three EMTs, including at least one intermediate EMT (EMT-I) or nurse per ambulance. Ambulance crew can provide care that is comparable to the EMT-I level in the US, including intravenous fluids, endotracheal intubation, or laryngeal mask airway insertion under direct medical oversight. However, they cannot use medications for ALS (e.g. epinephrine, amiodarone). This means that there is not enough time to provide ALS in the field and only BLS is being performed. One of the most important factors for ROSC is that high-quality BLS should be continuously performed for a reasonable period of time, and 10 minutes of on-site cardiopulmonary resuscitation (CPR) is a very short time.

\subsection{Selection of the study participants}

This study was performed on non-trauma cardiac arrest patients who were 18 years of age or older who underwent CRP. On the other hand, cardiac arrest patients due to trauma, poisoning, pregnancy, lack of resuscitation, and those whose CPRs were put off due to definite death (e.g., decapitation, incineration, decomposition, rigor motis, or dependent cyanosis) were excluded from this study. It should be mentioned that in case the patients were qualified for the pilot project but the patients' family did not agree on the SALS pilot project and wanted the patient to be transported to the hospital quickly, BLS was given as per standard procedure, and they were then excluded from the study.

\subsection{Intervention}

In the "Before" phase, the EMTs dispatched to the scene, performed a conventional BLS for about $5 \mathrm{~min}$, and transferred the patient to the nearest hospital. The EMTs, who could not declare death or stop CPR, could take medical directions from a physician via telephone when there was an apparent suspicion of death (e.g., decapitation, rigor mortis, decomposition, and dependent lividity). In the "After" phase, at the area implemented SALS pilot project, they were allowed to undergo further intravenous fluid, intravenous medications, and advanced airway insertion (e.g., I-gel and endotracheal intubation) under physician's direct medical oversight through video call.

\subsection{Outcome measure}

The first outcome measure was to assess the prehospital-spontaneous recovery rate of the hospital, and the second outcome measure included the estimation of the survival and neurological recovery rates according to the Utstein style (14). Neurological recovery rates were measured at discharge using the cerebral performance category (CPC) score. It is worth mentioning that CPCs 1 and 2 were defined as good neurological recovery (15).

\subsection{Statistical analysis}

Statistical analysis was performed using SPSS software (version 21.0) (SPSS Inc. Chicago, Illinois, USA), and nominal variables were expressed as counts and percentages of total numbers. Furthermore, continuous variables were expressed 
using median and interquartile range (IQR). Differences between BLS and SALS groups were compared using the chi-square and Fisher's exact tests for nominal variables and independent sample t-test for continuous variables. The statistical significance was judged to be the case when the pvalue was less than 0.05 . The propensity score was matched using a logistic regression model to compare the effects of SALS vs. BLS on the outcomes of OHCA patients. Data with missing values were excluded from the collected data. The independent variables (covariates) being potential risk factors of gender, age, witnessed, bystander CPR, public place, and shockable rhythm were included in this study. The propensity scores were calculated to a maximum of 10 decimal places. The patients who received SALS were matched to the closest BLS group patient whose propensity score differed by less than $1 / 10-4$. Adjusted multivariable logistic regression analysis was used to examine the associations between SALS and outcome, except for data with missing values. Associations were presented as odds ratios (ORs) with 95\% confidence intervals (CI).

\section{Results}

\subsection{Outcomes}

Among 1,333 EMS-assessed OHCA, 235 and 336 patients in the "Before" and "After" phases were included in the final analysis (Figure 2). In the "After" phase, SALS was performed on 198 patients (SALS group), and 138 patients received no SALS and were transported to the hospital (No SALS group). Table 1 provides patients' characteristics, EMS factor, time interval, post-resuscitation care, and outcomes for the overall group. In the SALS group, $69.7 \%$ of the bystanders provided the OHCA patients with CPR, whereas $56.6 \%$ of the bystanders provided the BLS group with CPR. A number of patients with shockable rhythm were more frequent in the SALS group, compared to the BLS group (20.4\% vs. $16.6 \%, \mathrm{P}<0.001)$, whereas the success rate of the defibrillation by the EMS provider were lower in the SALS group, compared to the BLS group ( $47.5 \%$ vs. $56.4 \%, \mathrm{P}=0.004$ ).

There was no difference between the two groups regarding the EMS response time and transport time intervals. Since ALS was implemented in the scene, from arrival at the scene to departure, the time interval increased from 9.0 (median, 4.0-10.0) min to 27.0 (median, 21.0-33.0) $\mathrm{min}(\mathrm{P}<0.001)$. On the other hand, the time interval of the EMS arrival to first ROSC was 22.0 (median, 14.0-37.0) min in the SALS group that was shorter than that in the BLS group (29.0 min; median, 18.0-46.0) $\min (\mathrm{P}<0.001)$. Prehospital ROSC rate in the SALS group was higher
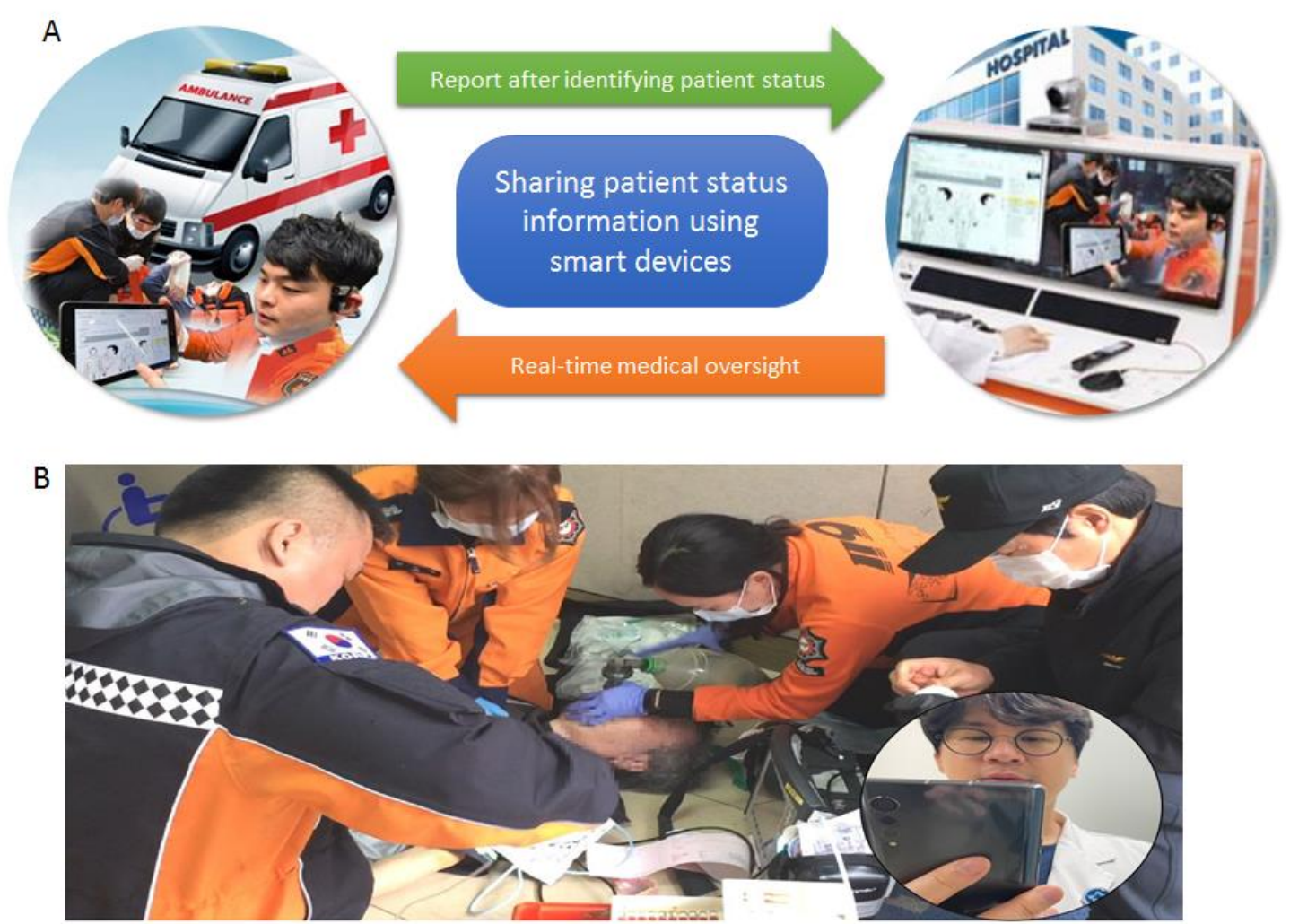

Figure 1. Graphic concept (A) and actual capture image (B) for smart advanced life support. The SALS is a field advanced resuscitation by paramedics with video communication based direct medical oversight. All medical oversights were performed by an emergency medical practitioner 


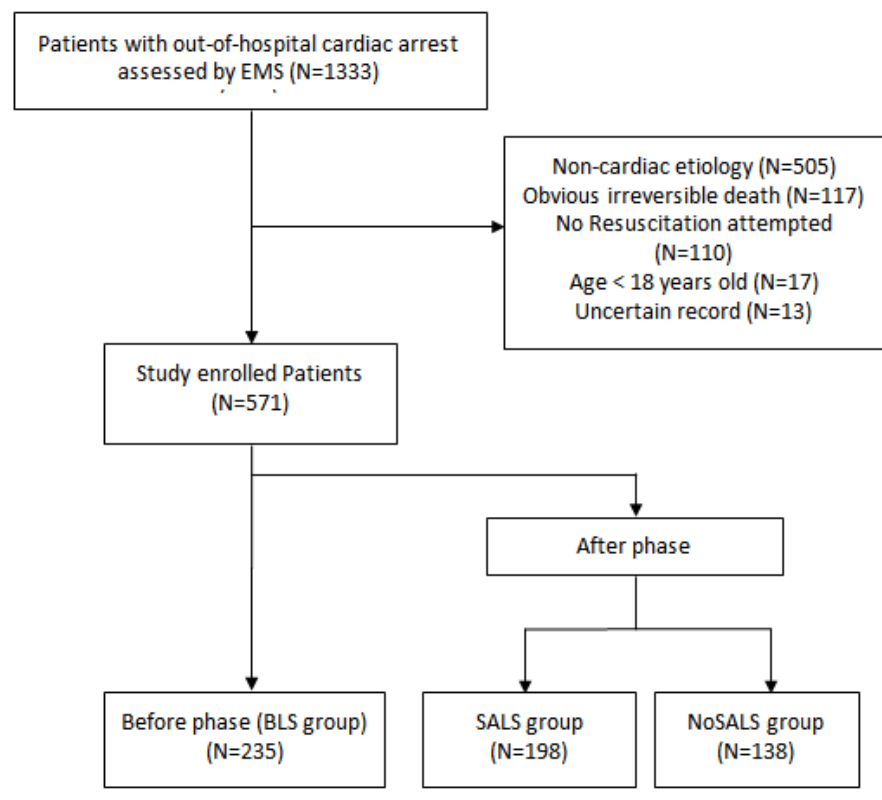

Figure 2. Study flowchart

EMS; emergency medical service, CPR; cardiopulmonary resuscitation, SALS; Smartphone videoassisted advanced life support

\begin{tabular}{|c|c|c|c|}
\hline Characteristics & $\begin{array}{c}\text { BLS group } \\
(\mathrm{N}=235)\end{array}$ & $\begin{array}{c}\text { SALS group } \\
(\mathrm{N}=198)\end{array}$ & P-Value \\
\hline \multicolumn{4}{|l|}{ Patient and Environmental factors } \\
\hline Gender (male)-No (\%) & $147(62.5)$ & $135(68.2)$ & $<0.001$ \\
\hline Age (year), median (IQR) & $70(56-80)$ & $71(56-79)$ & 0.48 \\
\hline Public place-No.(\%) & $40(17.3)$ & $35(17.6)$ & 0.55 \\
\hline Witnessed arrest-No.(\%) & $115(48.9)$ & $97(49.0)$ & 0.10 \\
\hline Bystander CPR-No.(\%) & $133(56.6)$ & $138(69.7)$ & $<0.001$ \\
\hline Initial cardiac rhythm-No.(\%) & & & $<0.001$ \\
\hline Ventricular fibrillation/tachycardia & $39(16.6)$ & $40(20.4)$ & \\
\hline Pulseless electrical activity & $43(18.4)$ & $33(16.7)$ & \\
\hline Asystole & $150(63.9)$ & $124(62.9)$ & \\
\hline Unknown & $3(1.3)$ & $1(0.1)$ & \\
\hline \multicolumn{4}{|l|}{ EMS factor } \\
\hline Defibrillation shock-No.(\%) & $58(24.7)$ & $63(31.8)$ & $<0.001$ \\
\hline EMS Defibrillation Success-No.(\%) & $30(56.4)$ & $25(47.5)$ & 0.004 \\
\hline Number of Defibrillation attempts-No. (SD) & $2.23(1.72)$ & $3.44(2.49)$ & $<0.001$ \\
\hline Prehospital advanced airway-No.(\%) & & $189(95.5)$ & NA \\
\hline \multicolumn{4}{|l|}{ Prehospital drug administered-No.(\%) } \\
\hline Epinephrine-No.(\%) & & $136(69.0)$ & NA \\
\hline Amiodarone-No.(\%) & & $11(5.6)$ & NA \\
\hline Fluid bolus-No.(\%) & & $146(73.7)$ & NA \\
\hline \multicolumn{4}{|l|}{ Time interval-min } \\
\hline Response time interval (Call to EMS arrival on scene)-median (IQR) & $8.0(6.0-11.0)$ & $7.0(6.0-9.0)$ & $<0.001$ \\
\hline From arrival at scene to departure-median (IQR) & $9.0(6.0-13.0)$ & $27.0(21.0-33.0)$ & $<0.001$ \\
\hline From departure to arrival at hospital-median (IQR) & $6.0(4.0-10.0)$ & $6.0(4.0-9.0)$ & 0.005 \\
\hline Call to arrival at hospital-median (IQR) & $25.0(20.0-32.0)$ & $42.0(35.0-49.0)$ & $<0.001$ \\
\hline Call to EMS basic life support-median (IQR) & $9.0(7.0-12.0)$ & $9.0(7.0-12.0)$ & $<0.001$ \\
\hline From arrival at scene to EMS basic life support-median (IQR) & $1.0(0-2.0)$ & $2.0(1.0-3.0)$ & 0.077 \\
\hline From arrival at scene to first ROSC-median (IQR) & $29.0(18.0-46.0)$ & $22.0(14.0-37.0)$ & $<0.001$ \\
\hline From EMS basic life support to first ROSC-median (IQR) & $28.0(16.0-47.0)$ & $20.0(12.0-35.0)$ & 0.02 \\
\hline Call to first ROSC-median (IQR) & $38.0(26.0-55.0)$ & $30.0(21.0-45.0)$ & $<0.001$ \\
\hline From arrival at scene to first shock delivered-median (IQR) & $4.0(2.0-8.0)$ & $3.0(2.0-4.0)$ & $<0.001$ \\
\hline \multicolumn{4}{|l|}{ Post resuscitation care } \\
\hline PCI-No.(\%) & $6(2.6)$ & $14(7.0)$ & $<0.001$ \\
\hline Hypothermia therapy-No.(\%) & $12(5.0)$ & $12(6.0)$ & 0.02 \\
\hline ECMO therapy-No.(\%) & $4(1.7)$ & $6(3.0)$ & $<0.001$ \\
\hline \multicolumn{4}{|l|}{ Outcome } \\
\hline Return of spontaneous circulation & $20(8.5)$ & $44(22.2)$ & $<0.001$ \\
\hline Admission to hospital & $52(22.1)$ & $37(18.6)$ & 0.008 \\
\hline Survival to hospital discharge & $16(6.8)$ & $20(10.1)$ & $<0.001$ \\
\hline Good neurologic recovery & $10(4.2)$ & $13(6.6)$ & $<0.001$ \\
\hline
\end{tabular}

IQR, interquartile range; CPR, cardiopulmonary resuscitation; EMS, emergency medical service; SD, standard deviation; NA, not applicable; ROSC, return of spontaneous circulation; PCI, percutaneous coronary intervention; ECMO, extracorporeal membrane oxygenation. 
than that in the BLS group ( $n=442 ; 22.2 \%$ vs. $n=200$; 8.5\%; $\mathrm{P}<0.001$, respectively). In the SALS group, 20 $(10.1 \%)$ patients survived hospital discharge, compared to $16(6.8 \%)$ cases in the BLS group $(\mathrm{P}<0.001)$. Furthermore, the rates of survival with favorable neurologic status were $13(6.6 \%)$ and 10 (4.2\%) cases in the SALS and BLS groups $(\mathrm{P}<0.001)$, respectively.

\subsection{Logistic regression analysis}

Table 2 presents the multivariable logistic regression analysis on the outcomes for the SALS group, compared to the BLS group. The utilization of the SALS protocol was associated with an increased likelihood of prehospital ROSC in analysis (22.2 vs. 8.5, adjusted OR: 4.04; $\mathrm{P}<0.001$ ), survival to discharge (10.1 vs. 6.8 , adjusted OR: 1.23; $\mathrm{P}<0.001$ ), and good neurologic recovery (6.6 vs. 4.2 , adjusted OR: 1.39; $\mathrm{P}<0.001$ ).

\subsection{Propensity score matching analysis}

In total, 304 patients were matched on the propensity score for each BLS and SALS group. Baseline characteristics comparing the propensitymatched BLS and SALS receiving patients are shown in Table 3. The patients who received SALS were associated with an increased odd of prehospital ROSC

\begin{tabular}{|c|c|c|c|c|c|}
\hline Outcome & $\begin{array}{c}\text { BLS group } \\
(\mathrm{N}=235)\end{array}$ & $\begin{array}{c}\text { SALS group } \\
(\mathrm{N}=198)\end{array}$ & P-Value & $\begin{array}{c}\text { OR } \\
(95 \% \mathrm{CI})\end{array}$ & $\begin{array}{c}\text { *Adjusted OR } \\
\text { (95\% CI) }\end{array}$ \\
\hline Prehospital ROSC-No.(\%) & $20(8.5)$ & $44(22.2)$ & $<0.001$ & $\begin{array}{c}3.08 \\
(2.57-3.68)\end{array}$ & $\begin{array}{c}4.04 \\
(3.24-5.03)\end{array}$ \\
\hline †ED ROSC-No.(\%) & $63(26.8)$ & $16(8.0)$ & $<0.001$ & $\begin{array}{c}0.24 \\
(0.20-0.29)\end{array}$ & $\begin{array}{c}0.22 \\
(0.18-0.27)\end{array}$ \\
\hline ‡Total ROSC-No.(\%) & $83(35.3)$ & $60(30.3)$ & $<0.001$ & $\begin{array}{c}0.79 \\
(0.70-0.90)\end{array}$ & $\begin{array}{c}0.73 \\
(0.63-0.85)\end{array}$ \\
\hline Admission to hospital-No.(\%) & $52(22.1)$ & $37(18.6)$ & 0.008 & $\begin{array}{c}0.82 \\
(0.70-0.95)\end{array}$ & $\begin{array}{c}0.70 \\
(0.58-0.83)\end{array}$ \\
\hline Survival to discharge-No.(\%) & $16(6.8)$ & $20(10.1)$ & $<0.001$ & $\begin{array}{c}1.48 \\
(1.20-1.84)\end{array}$ & $\begin{array}{c}1.23 \\
(0.96-1.68)\end{array}$ \\
\hline Good neurologic recovery-No.(\%) & $10(4.2)$ & $13(6.6)$ & $<0.001$ & $\begin{array}{c}1.69 \\
(1.29-2.21)\end{array}$ & $\begin{array}{c}1.39 \\
(1.00-1.92)\end{array}$ \\
\hline
\end{tabular}

OR, odds ratio; CI, confidence interval; ROSC, return of spontaneous circulation; ED, emergency department;

* The analysis is adjusted for gender, age, place, witness, bystander CPR, initial shockable rhythm, response time interval (call to EMS arrival on scene).

† ED ROSC is ROSC, documented initially in the emergency department without prehospital ROSC.

‡ Total ROSC corresponds to obtaining prehospital ROSC or ED ROSC.

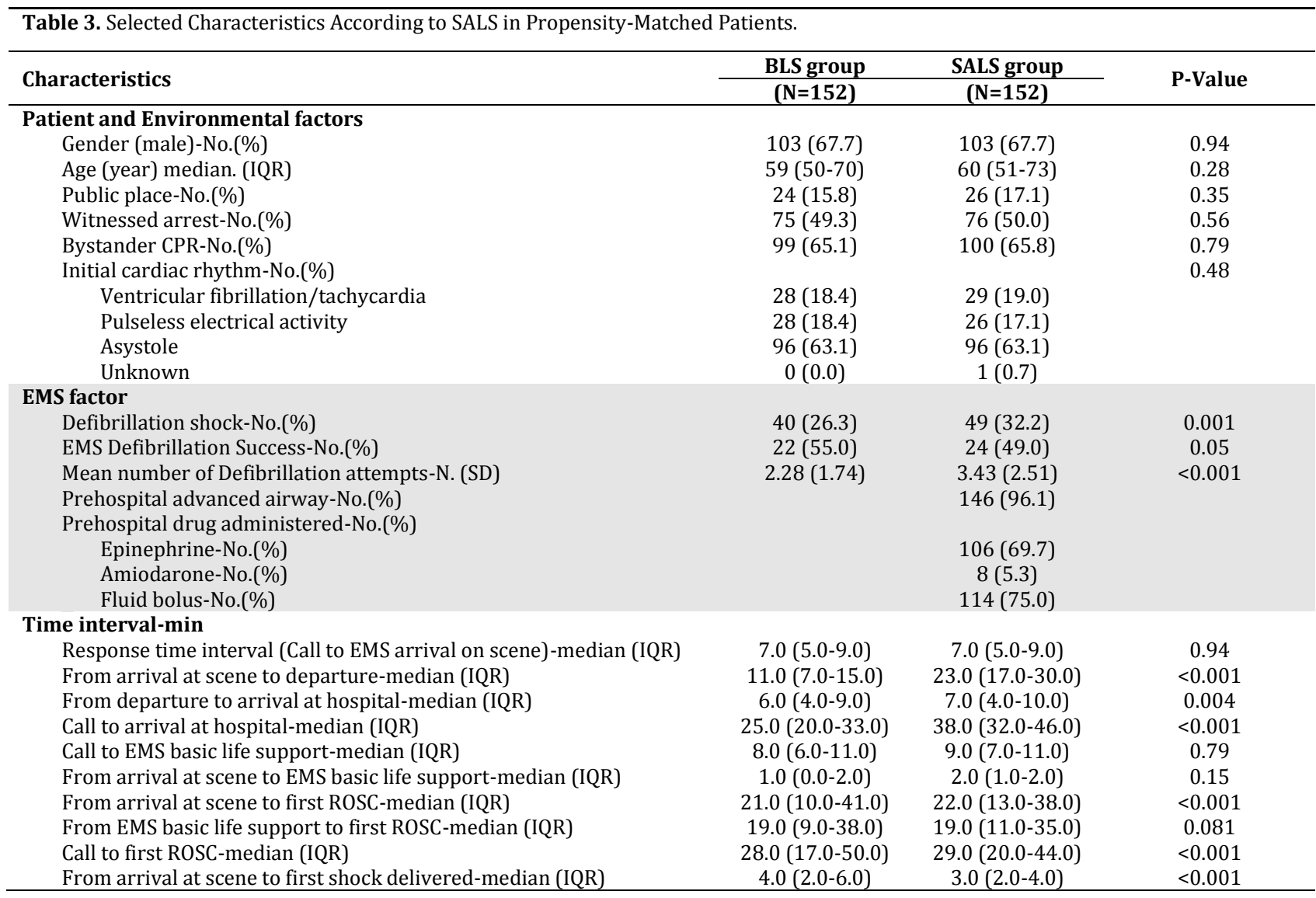




\begin{tabular}{|c|c|c|c|}
\hline \multicolumn{4}{|l|}{ Post resuscitation care } \\
\hline PCI-No.(\%) & $4(2.6)$ & $5(3.2)$ & 0.02 \\
\hline Hypothermia therapy-No.(\%) & $8(5.2)$ & $9(5.9)$ & 0.09 \\
\hline ECMO therapy-No.(\%) & $1(0.6)$ & $3(2.0)$ & $<0.001$ \\
\hline \multicolumn{4}{|l|}{ Outcome } \\
\hline Return of spontaneous circulation & $13(8.5)$ & $33(21.7)$ & $<0.001$ \\
\hline Admission to hospital & $32(21.0)$ & $28(18.4)$ & 0.02 \\
\hline Survival to hospital discharge & $11(7.2)$ & $14(9.2)$ & 0.06 \\
\hline Good neurologic recovery & $6(3.9)$ & $9(5.9)$ & 0.008 \\
\hline
\end{tabular}

IQR, interquartile range; CPR, cardiopulmonary resuscitation; EMS, emergency medical service; SD, standard deviation; NA, not applicable; ROSC, return of spontaneous circulation; PCI, percutaneous coronary intervention; ECMO, extracorporeal membrane oxygenation.

\begin{tabular}{|c|c|c|c|c|c|c|c|c|}
\hline \multirow{2}{*}{$\begin{array}{l}\text { Model } \\
\text { SALS(0) vs. BLS(1) } \\
(n=3,042)\end{array}$} & \multicolumn{2}{|c|}{ Prehospital ROSC } & \multicolumn{2}{|c|}{ Survival to admission } & \multicolumn{2}{|c|}{ Survival to discharge } & \multicolumn{2}{|c|}{$\begin{array}{l}\text { Good neurologic } \\
\text { recovery }\end{array}$} \\
\hline & $\mathrm{OR}(95 \% \mathrm{CI})$ & P-Value & OR(95\% CI $)$ & P-Value & OR $(95 \% \mathrm{CI})$ & P-Value & OR $(95 \% \mathrm{CI})$ & P-Value \\
\hline Unadjusted & $\begin{array}{c}2.96 \\
(2.39-3.68)\end{array}$ & $<0.001$ & $\begin{array}{c}0.81 \\
(0.68-0.97)\end{array}$ & 0.02 & $\begin{array}{c}1.29 \\
(1.00-1.67)\end{array}$ & 0.05 & $\begin{array}{c}1.54 \\
(1.12-2.13)\end{array}$ & 0.008 \\
\hline Adjusted for propensity & $\begin{array}{c}3.08 \\
(2.47-3.84)\end{array}$ & $<0.001$ & $\begin{array}{c}0.81 \\
(0.67-0.97)\end{array}$ & 0.02 & $\begin{array}{c}1.31 \\
(1.00-1.70)\end{array}$ & 0.05 & $\begin{array}{c}1.61 \\
(1.15-2.25)\end{array}$ & 0.006 \\
\hline $\begin{array}{l}\text { Adjusted for propensity } \\
\text { and selected variables* }\end{array}$ & $\begin{array}{c}3.60 \\
(2.83-4.58)\end{array}$ & $<0.001$ & $\begin{array}{c}0.77 \\
(0.63-0.93)\end{array}$ & 0.007 & $\begin{array}{c}1.33 \\
(1.00-1.77)\end{array}$ & 0.05 & $\begin{array}{c}1.73 \\
(1.19-2.53)\end{array}$ & 0.004 \\
\hline
\end{tabular}

ROSC, return of spontaneous circulation; OR, odds ratio; $\mathrm{CI}$, confidence interval;

* The analysis is adjusted for gender, age, place, witness, bystander CPR, initial shockable rhythm, response time interval (call to EMS arrival on scene).

(OR: 3.60; 95\% CI: 2.83-4.58; $\mathrm{P}<0.001$ ), survival to discharge (OR: 1.33, 95\% CI: 1.00-1.77; P=0.05), good neurologic recovery (OR: 1.73, 95\% CI: 1.19-2.53; $\mathrm{P}=0.004$ ) (Table 4).

\section{Discussion}

This study was the first attempt to clarify that the Advanced Cardiac Life Support (ACLS) performed by visual direct medical direction using smartphones was effective for the ROSC and neurological recovery of the OHCA patients. Furthermore, an unusual result showed improvement in the resuscitation and neurological recovery rateS by implementing ALS for the OHCA patients. Although the effect of prehospital ALS in the OHCA has been questioned recently, the Spanish OHCA registry reported a higher rate of survival with the favorable neurologic outcomes of physicians who treated the OHCA patients, compared to paramedic-based EMS legions (16). This study showed that recent developments in smartphone technology may be integrated with ALS.

Recently, the development of information and communication technology has made various attempts in the field of EMS. The use of smartphones for medical oversight in cardiac arrest situations is very useful. Smartphones are lightweight, portable, easy to use, and cheaper than other medical devices. These advantages make it easier for the EMTs and oversight doctors to share large amounts of information in the emergency. There are several reasons why this study could produce a good result in a short period of time. First, it is the role of the oversight physician to emphasize high-quality CPR. Until recently, limited studies have indicated that exposure to the OHCA for individual paramedics is rare. Dyson et al. reported that paramedics in Australia treated only 1.4 OHCA patients per year (17). Such low exposure may impact the paramedics' ability to perform resuscitation skills according to the guidelines, which is vital to patient survival (18).

Despite many controversies, it has been reported that physicians provide benefits to patients who have cardiac arrest before the hospital (19). Physicians are able to carry out advanced procedures, such as airway management and epinephrine use, adhere to treatment according to guidelines, and have the latest knowledge. However, in this study, the physician did not respond to the field; therefore, the latter may have affected the outcome of the treatment. The physician advises and instructs on-site EMT leaders on team management, detailed ECG analysis, the importance of BLS, and accurate ventilation. The presence of a physician with such experience and knowledge helps to improve the quality of care of the CPR team. In addition, EMT team leaders receiving continuous feedback could learn oversight during resuscitation and grow into ACLS experts. In addition to the direct instruction of the physician, the Hawthorne effect may also have worked (20).

Furthermore, the introduction of a strategy in each director can be assumed to have influenced performance (21-23). Each month, a committee meeting is held with oversight physicians, university hospital emergency attending doctors, fire office education administrators, and dispatcher quality managers in each area. Following that, the monthly results are shared, and problems are discovered and corrected. Based on the results of the committee meeting, re-training is conducted, transfer hospitals 
are selected, and hospitals cooperate to provide effective intervention after cardiac arrest. This process provides an opportunity to objectively identify and rectify the field weaknesses.

Lastly, it is also meaningful that each oversight doctor and EMT tries to communicate through social networking service (SNS). It is difficult for the EMTs and oversight doctors to make contact with video or voice only in a cardiac arrest situation because it is much more office-friendly. Personal exchange among people increases intimacy. This affects feedback and retraining effects. Communication channels between hospitals and paramedics have indirectly positive effects on teamwork.

As a result of this intervention, pre-hospital ROSC was increased in the SALS group; however, the survival hospitalization decreased further. On the other hand, survival and neurological recovery rates were found to be higher in the SALS group. The time to ROSC is a significant prognostic factor for the survival and neurological recovery rates of comatose OHCA patients $(24,25)$. The ROSC of the BLS group has been performed mostly in hospitals; however, the time to ROSC was longer in that group. On the other hand, the ROSC of the SALS group was mostly performed in the field and the time to ROSC was shorter. The prognosis of the OHCA patients with rearrest was poor (26). Furthermore, the hospital admission rate of the SALS group was further reduced due to the inability to carry out critical interventions during transport, and rearrest occurred after arrival at the hospital. Nonetheless, the decline in the time to the ROSC seems to increase survival and neurological recovery rates.

\section{Limitations}

Although this study paved the way for prehospital ACLS, it suffers from some limitations. First, it is a controlled "Before-After" study, not a planned randomized controlled trial (RCT). In fact, the outcome of cardiac arrest in Korea is improving every year. The RCT is required to reflect the local demographic, social, and regional characteristics. In Korea, the RCT for cardiac arrest patients is ethically problematic and various legal aspects are needed. Moreover, due to the nature of this pilot project conducted by the government, it was not possible to determine patients who would randomly perform SALS. However, according to the results of the cardiac arrest in Korea, the rate of survival discharge from 2006 to 2016 increased by an average of $0.54 \%$ from $2.3 \%$ to $7.6 \%$, and the neurological recovery rate increased by $0.36 \%$ from $0.6 \%$ to $4.2 \%$ each year. Furthermore, approximately, $40 \%$ of patients were excluded from the study due to their families' unwillingness. This can result in another selection bias, which can lead to confusion in a variety of other variables. However, there is no doubt about this result. To solve this problem, our results were statistically corrected using the propensity score matching method. In addition, there were various changes, such as video medical direction, change to ACLS, increase of on-site time, drug administration, localization model, double tier dispatch, Hawthorne effect, use of SNS, and training of the EMTs; however, it was not possible to measure the effects of each of them. Nonetheless, since pre-hospital ALS and direct medical guidance for the OHCA patients are intertwined, it is difficult to measure the parameters separately. Additionally, the subjective evaluation involvement was complex. The authors considered these changes to be the basic prerequisites for video medical direction and ALS. Accordingly, further studies are required to analyze the effect of each variable.

\section{Acknowledgments}

The SALS protocol was funded by the Ministry of Health and Welfare Department of Korea and supported by the National Emergency Medical center and the Central fire agency. The authors specially appreciate the contribution of all paramedics and emergency physicians who involved in this protocol.

\section{Footnotes}

Authors' Contribution: YP contributed to the creation of the study material, participated in the design of the study, drafted and performed the statistical analyses. SHJ participated in the design of the study and helped to revise the manuscript. All authors read and approved the final manuscript and take full responsibility for all aspects of the study.

Conflict of Interests: The authors declare that they have no competing interests regarding the publication of the present study.

Ethical Approval: This study was conducted after the Institutional Review Board's approval from the Research Ethics Committee of the Chosun University Hospital, Gwangju, South Korea.

Funding/Support: This study was supported by a research fund from the Chosun University Hospital, Gwangju, South Korea (2014).

Financial Disclosure: The authors declare that there is no financial disclosure.

Informed consent: Informed consent was not required to conduct this study.

\section{References}

1. Lee MJ. Incidence and outcome of cardiac arrest in Korea. $J$ Korean Soc Emerg Med. 2012;23(2):168-80.

2. Yang HJ, Kim GW, Kim H, Cho JS, Rho TH, Yoon HD, et al. Epidemiology and outcomes in out-of-hospital cardiac arrest: a report from the NEDIS-based cardiac arrest registry in Korea. J Korean Med Sci. 2015;30(1):95-103. doi: 10.3346/jkms.2015.30.1.95. [PubMed: 25552889].

3. Ahn KO, Do Shin S, Suh GJ, Cha WC, Song KJ, Kim SJ, et al. 
Epidemiology and outcomes from non-traumatic out-ofhospital cardiac arrest in Korea: a nationwide observational study. Resuscitation. 2010;81(8):974-81. doi: 10.1016/j. resuscitation.2010.02.029. [PubMed: 20605312].

4. Berdowski J, Berg RA, Tijssen JG, Koster RW. Global incidences of out-of-hospital cardiac arrest and survival rates: systematic review of 67 prospective studies. Resuscitation. 2010; 81(11):1479-87. doi: 10.1016/j.resuscitation.2010.08.006. [PubMed: 20828914].

5. Ma MH, Ong ME, Shin SD, Siddiqui FJ, Tanaka H, Nishiuchi T, et al. The pan Asian resuscitation outcomes study (PAROS): characteristics of patients with out-of-hospital cardiac arrest across 7 countries in Asia. Circulation. 2014;130(Suppl 2):A69.

6. Sanghavi P, Jena AB, Newhouse JP, Zaslavsky AM. Outcomes after out-of-hospital cardiac arrest treated by basic vs advanced life support. JAMA Intern Med. 2015;175(2):196204. doi: 10.1001/jamainternmed.2014.5420. [PubMed: 25419698].

7. von Vopelius-Feldt J, Brandling J, Benger J. Systematic review of the effectiveness of prehospital critical care following outof-hospital cardiac arrest. Resuscitation. 2017;114:40-6. doi: 10.1016/j.resuscitation.2017.02.018. [PubMed: 28253479].

8. Stiell IG, Wells GA, Field B, Spaite DW, Nesbitt LP, De Maio VJ, et al. Advanced cardiac life support in out-of-hospital cardiac arrest. $N$ Engl J Med. 2004;351(7):647-56. doi: 10.1056/NEJMoa040325.

9. Sanghavi P, Jena AB, Newhouse JP, Zaslavsky AM. Outcomes of basic versus advanced life support for out-of-hospital medical emergencies. Ann Intern Med. 2015;163(9):681-90. doi: 10.7326/M15-0557. [PubMed: 26457627].

10. Cournoyer A, Notebaert É, Iseppon M, Cossette S, Londei-Leduc L, Lamarche $\mathrm{Y}$, et al. Prehospital advanced cardiac life support for out-of-hospital cardiac arrest: a cohort study. Acad Emerg Med. 2017;24(9):1100-9. doi: 10.1111/acem.13246. [PubMed: 28646584].

11. Hagihara A, Hasegawa M, Abe T, Nagata T, Nabeshima Y. Physician presence in an ambulance car is associated with increased survival in out-of-hospital cardiac arrest: a prospective cohort analysis. PLoS One. 2014;9(1):e84424. doi: 10.1371/journal.pone.0084424. [PubMed: 24416232].

12. Hamilton A, Steinmetz J, Wissenberg M, Torp-Pedersen C, Lippert FK, Hove L, et al. Association between prehospital physician involvement and survival after out-of-hospital cardiac arrest: a Danish nationwide observational study. Resuscitation. 2016;108:95-101. doi: 10.1016/j.resuscitation.2016.08.007. [PubMed: 27565859].

13. Böttiger BW, Bernhard M, Knapp J, Nagele P. Influence of EMS-physician presence on survival after out-of-hospital cardiopulmonary resuscitation: systematic review and metaanalysis. Crit Care. 2015;20(1):4. doi: 10.1186/s13054-0151156-6. [PubMed: 26747085].

14. Cummins RO, Chamberlain DA, Abramson NS, Allen M, Baskett PJ, Becker L, et al. Recommended guidelines for uniform reporting of data from out-of-hospital cardiac arrest: the Utstein Style. A statement for health professionals from a task force of the American Heart Association, the European Resuscitation Council, the Heart and Stroke Foundation of Canada, and the Australian Resuscitation Council. Circulation. 1991;84(2):960-
75. doi: 10.1161/01.cir.84.2.960. [PubMed: 1860248].

15. Rittenberger JC, Raina K, Holm MB, Kim YJ, Callaway CW. Association between cerebral performance category, modified rankin scale, and discharge disposition after cardiac arrest. Resuscitation. 2011;82(8):1036-40. doi: 10.1016/j.resuscitation.2011.03.034. [PubMed: 21524837].

16. Rosell-Ortiz F, Escalada-Roig X, del Valle PF, Sánchez-Santos L, Navalpotro-Pascual JM, Echarri-Sucunza A, et al. Out-ofhospital cardiac arrest (OHCA) attended by mobile emergency teams with a physician on board. Results of the Spanish OHCA Registry (OSHCAR). Resuscitation. 2017; 113:90-5. doi: 10.1016/j.resuscitation.2017.01.029. [PubMed: 28202420].

17. Dyson K, Bray JE, Smith K, Bernard S, Straney L, Finn J. Paramedic exposure to out-of-hospital cardiac arrest resuscitation is associated with patient survival. Circ Cardiovasc Qual Outcomes. 2016;9(2):154-60. doi: 10.1161/CIRCOUTCOMES.115.002317. [PubMed: 26812932].

18. Gold LS, Eisenberg MS. The effect of paramedic experience on survival from cardiac arrest. Prehosp Emerg Care. 2009; 13(3):341-4. doi: 10.1080/10903120902935389. [PubMed: 19499471].

19. Ohshige K, Shimazaki S, Hirasawa H, Nakamura M, Kin H, Fujii C, et al. Evaluation of out-of-hospital cardiopulmonary resuscitation with resuscitative drugs: a prospective comparative study in Japan. Resuscitation. 2005;66(1):53-61. doi: 10.1016/j.resuscitation.2004.10.019. [PubMed: 15993730].

20. McCarney R, Warner J, Iliffe S, Van Haselen R, Griffin M, Fisher P. The Hawthorne Effect: a randomised, controlled trial. BMC Med Res Methodol. 2007;7(1):30. doi: 10.1186/1471-2288-7-30. [PubMed: 17608932].

21. Kocher KE, Sklar DP, Mehrotra A, Tayal VS, Gausche-Hill M, Myles Riner R. Categorization, designation, and regionalization of emergency care: definitions, a conceptual framework, and future challenges. Acad Emerg Med. 2010; 17(12):1306-11. doi: 10.1111/j.1553-2712.2010.00932.x. [PubMed: 21122012].

22. Drennan IR, Verbeek PR. The role of EMS in regionalized systems of care. CJEM. 2015;17(4):468-74. doi: 10.1017/cem.2015.38. [PubMed: 26073708].

23. Cone DC, Brooke Lerner E, Band RA, Renjilian C, Bobrow BJ, Crawford Mechem C, et al. Prehospital care and new models of regionalization. Acad Emerg Med. 2010;17(12):1337-45. doi: 10.1111/j.1553-2712.2010.00935.x. [PubMed: 21122016].

24. Martinell L, Nielsen N, Herlitz J, Karlsson T, Horn J, Wise MP, et al. Early predictors of poor outcome after out-of-hospital cardiac arrest. Crit Care. 2017;21(1):96. doi: 10.1186/ s13054-017-1677-2. [PubMed: 28410590].

25. Kjaergaard J, Nielsen N, Winther-Jensen M, Wanscher M, Pellis T, Kuiper $\mathrm{M}$, et al. Impact of time to return of spontaneous circulation on neuroprotective effect of targeted temperature management at 33 or 36 degrees in comatose survivors of out-of hospital cardiac arrest. Resuscitation. 2015;96:310-6. doi: 10.1016/j.resuscitation.2015.06.021. [PubMed: 26159609].

26. Salcido DD, Sundermann ML, Koller AC, Menegazzi JJ. Incidence and outcomes of rearrest following out-ofhospital cardiac arrest. Resuscitation. 2015;86:19-24. doi: 10.1016/j.resuscitation.2014.10.011. [PubMed: 25447433]. 